\title{
Collapse Mechanisms of Lung Surfactant Protein DPPG
}

\author{
T.F. Alig, ${ }^{*}$ H.E. Warriner, ${ }^{* *}$ J.A. Zasadzinski* \\ *University of California Santa Barbara, Chemical Engineering Dept., Santa Barbara, CA 93106 \\ ** University of Pittsburgh, Dept. of Chemistry, Pittsburgh, PA 15260
}

Lung surfactant plays a crucial role in respiration [1]. Respiratory distress syndrome (RDS) can be caused by the lack of lung surfactant and can lead to death in premature infants $[1,2]$. Artificial lung surfactants are being developed to combat RDS, but a fundamental understanding of monolayer collapse is needed to properly design these artificial mixtures. This paper addresses the issue of monolayer collapse at high surface pressures and subsequent respreading. Monolayer collapse has been extensively studied, yet little is known about mechanisms of collapse and respreading [3-5]

Dipamitoylphosphatidylglycerol (DPPG) is a major component of lung surfactant and is a charged lipid at the studied $\mathrm{pH}=7$. Using a Langmuir trough, isotherms of DPPG were obtained at $30^{\circ} \mathrm{C}$ on varying subphase salt concentrations and analyzed to determine the amount of collapse material that reincorporates into the monolayer upon expansion. The recovery of a monolayer is defined by the amount of material that reincorporates into the monolayer divided by the amount of material that was collapsed into the subphase. It was discovered that as the subphase salt concentration increased from $0 \mathrm{M}$ to $1 \mathrm{M} \mathrm{NaCl}$ the recovery increased from $28 \%$ to $95 \%$. In fact the recovery was found to be directly proportional to the inverse Debye length. This data clearly shows that electrostatic forces have a profound effect on the monolayer recovery.

The monolayer was also studied by Brewster Angle Microscopy (BAM). The BAM images give a sense of monolayer morphology at a relatively large scale (figure 1). The increase in subphase salt causes a more cluttered field of view in the BAM images. Unfortunately the objects of interest are smaller than the resolution of the BAM so the images show little about the collapse structures. However, the BAM demonstrates that as the subphase salt concentration is increased more collapse material stays near the surface, showing up as specks in the images.

Atomic Force Microscopy (AFM) was used to study the collapse structure at a smaller scale. The collapse structures were deposited onto a mica substrate using the Langmuir-Blodgett technique and then imaged with the AFM (figure 2). The AFM revealed that the collapse structure is the same regardless of the subphase salt concentration. Although the large scale images show vastly different morphologies, when the scale is decreased the images reveal that the basic unit is a vesicle in all cases. The difference is where the vesicles go after they bud off from the monolayer.

When no salt is present, electrostatic forces push the vesicles away from the surface, resulting in a relatively smooth AFM image. However, when the vesicles are freely floating away from the monolayer they are attracted to the mica substrate and this results in large clumps of vesicles on some parts of the mica substrate. When the subphase contains $150 \mathrm{mM} \mathrm{NaCl}$, the electrostatic repulsion is somewhat screened and all area of the monolayer have some vesicles nearby. Still however some of the freely floating vesicles are attracted to the mica and result in large clumps. The most interesting images come from the $1 \mathrm{M} \mathrm{NaCl}$ case. When the salt concentration is high, the electrostatic repulsion is almost completely screened and the Van der Waals forces are dominating. 
This results in the vesicles remaining very near where they left the monolayer. The high salt AFM images have a snakeskin like structure that demonstrates this effect quite clearly. As the monolayer is compressed, solid domains are formed and pushed together. Eventually material breaks off from the edges of the solid domains and form vesicles which stay near the domain edge.

It has been found that for DPPG the reason for increased monolayer recovery with increasing subphase salt concentration is not a different collapse structure, but rather where the lipids are stored. The high salt subphase allow the electrostatics to be screened and the lipids remain near the monolayer where they can more easily reincorporate into the monolayer [6].

References

[1] J. Goerke, Biochim. Biophys. Acta 1408, 79 (1998)

[2] J.A. Clements, Physiologist 5, 11 (1962)

[3] C. Ybert et al., J. Phys.: Condens. Matter 14, 4753 (2002)

[4] H. Diamant et al., Phys. Rev. E 63, 061602 (2001)

[5] A. Gopal and K.Y.C. Lee, J. Phys. Chem. B 105, 10348 (2001)

[6] A special thanks to Lily Lee and Tina Duran of UCSB for their aid in this work.

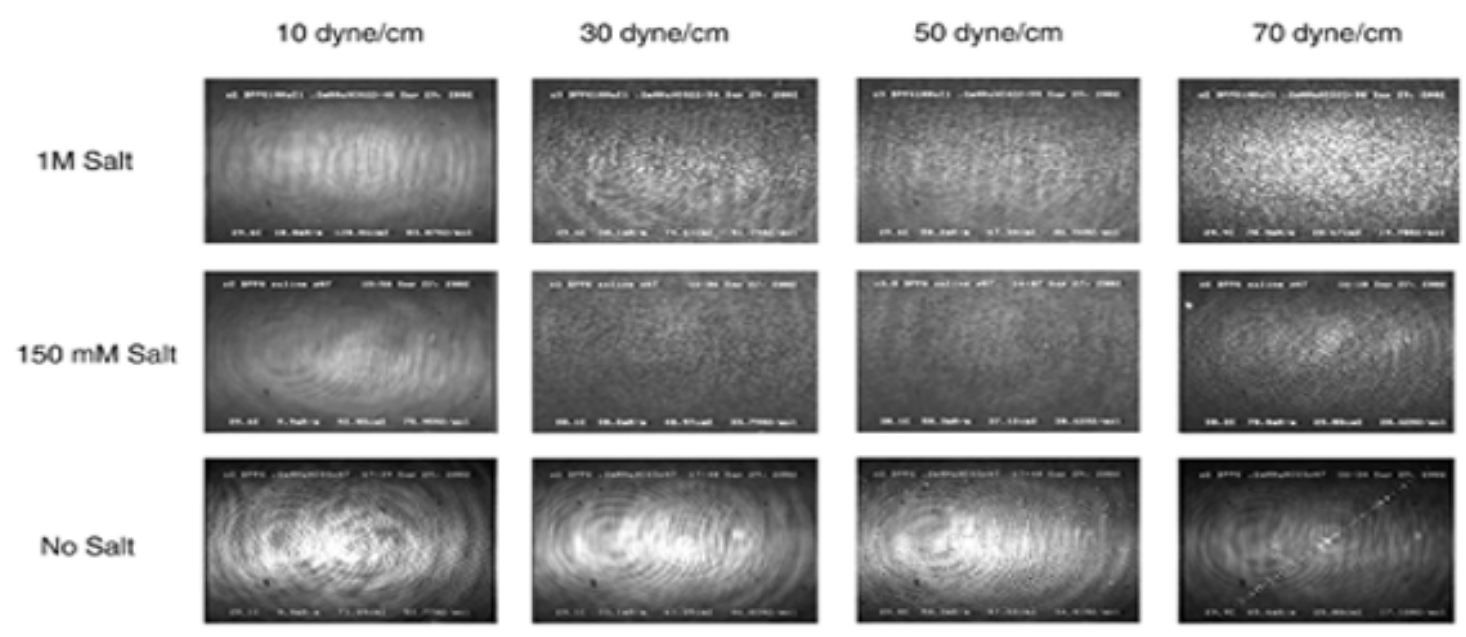

Figure 1. BAM images of DPPG monolayer during compression. Images are 1300x1425 $\mu$ m.
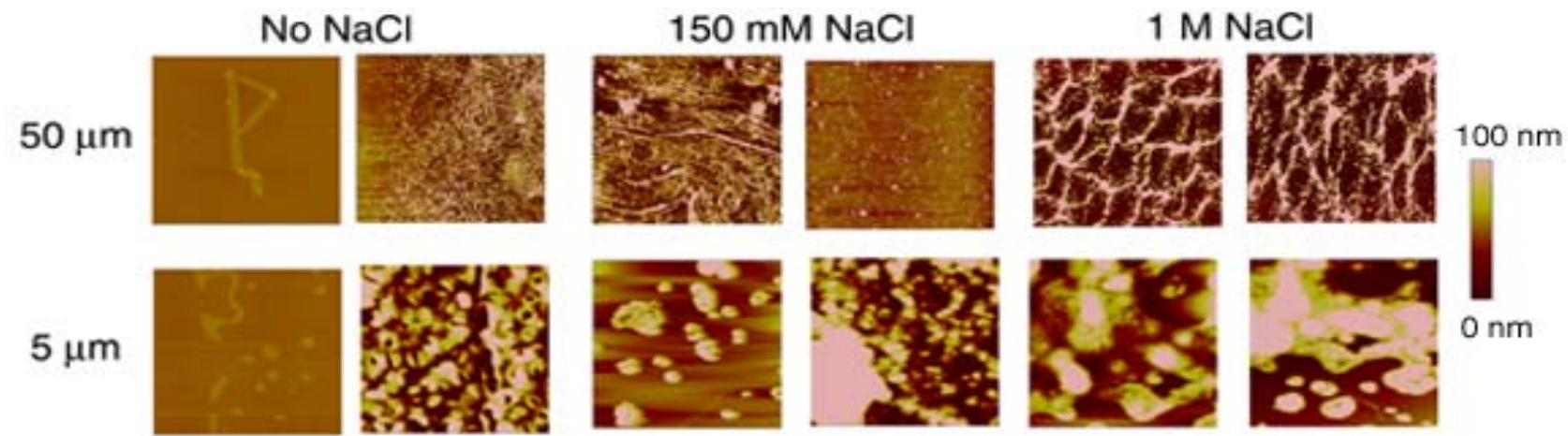

Figure 2. AFM images of collapse structures. Top row images are 50x50 $\mu \mathrm{m}$, bottom are $5 \times 5 \mu \mathrm{m}$. 